\title{
Optimizing Performance in Return to Play After Sport-Related Concussion in Elite Ice Hockey Players: A Sports Physical Therapy and Athletic Trainer Perspective
}

\author{
Mario Bizzini, PT, PhD $^{1}$ a \\ 1 Human Performance Lab and Swiss Concussion Centre, Schulthess Clinic; Swiss Sports Physiotherapy Association \\ Keywords: sport-related concussion, return to play, ice hockey \\ https://doi.org/10.26603/001c.31953
}

International Journal of Sports Physical Therapy

Vol. 17, Issue 2, 2022

\section{INTRODUCTION}

In the last two decades, sport-related concussion (SRC) has received growing attention within the sporting world. High SRC incidence rates have been reported for contact team sports such as rugby, American football and ice hockey, whereas men's football (soccer) is associated with the lowest concussion incidence. ${ }^{1}$ Furthermore, higher rates of SRC were found in female football and ice hockey players during competition and training.

Since 2017, the Concussion in Sport Group (CISG) consensus statement has set the benchmark for physicians, athletic trainers, sports physical therapists and other healthcare providers involved in athlete care at any level of sport. ${ }^{2}$ Sport-related concussion is defined as a subset of mild traumatic brain injury without structural anomalies on conventional neuroimaging. Headache and dizziness are the most common acute symptoms of SRC that usually resolve after 7 to 10 days, although 10\% to $15 \%$ of symptoms may persist for a longer period. The CISG statement describes 11 "Rs" of clinical SRC management: recognize, remove, re-evaluate, rest, rehabilitation, refer, recover, return to sport, reconsider, residual effects and sequelae, and risk reduction. ${ }^{2}$ After an initial period of 24 to 48 hours of physical and cognitive rest, a gradual return to sport (RTS) strategy is recommended.

Feddermann-Demont et al. ${ }^{3}$ recently presented a systematic approach for the initial examination, diagnosis, and management after SRC for high-level football (soccer) players, which includes a detailed RTS program. To date, this procedure is the only example of a sport-specific comprehensive strategy available for team physicians and healthcare personnel to treat SRC athletes.

In elite ice hockey, concussion protocols of the National Hockey League (NHL) ${ }^{4}$ and International Ice Hockey Federation (IIHF) ${ }^{5}$ exist and are consistent with the CISG consensus and RTS guidelines. While the CISG-RTS guideline entails generic activity descriptions (Table 1), the NHL protocol provides an example of a graded return to play progression for ice hockey players after SRC.

This clinical commentary presents a detailed RTS program for high-level ice hockey, which is based on the most recent recommendations as well as clinical and practical experience. The proposed comprehensive protocol specifically aims to optimize performance in elite male and female ice hockey players after SRC.

\section{RETURN TO SPORT}

While the team physician (or designee) supervises the RTS protocol, ${ }^{2,3}$ sports physical therapists, athletic trainers, and conditioning coaches closely cooperate to monitor the injured player throughout the various RTS stages.

Based on CISG guidelines, SRC players are progressively exposed to training activities (known as graduated RTS progression) that do not provoke or worsen symptoms (Table 1). There should be a period of at least 24 hours (or longer) for each progression step. If any symptoms worsen during exercise, the athlete should return to the previous stage. ${ }^{2}$ The process from one phase to the next should be individualized based on the current signs and symptoms, and on the unique characteristics (i.e. age, experience and skills) of each player.

The 2016 consensus statement on RTS defines three elements of the "RTS continuum": return to participation, return to sport and return to performance. ${ }^{6}$ While the ultimate aim of returning to pre-injury performance level is ideally reached after complying with the consensus RTS process, the goal of the supporting staff is to safely and optimally guide the athlete through the "training to perform" regimen. In a recent editorial, Reinhold ${ }^{7}$ highlighted that sports physical therapy should aim to optimize performance: the key concept (or the "performance spectrum continuum") not only involves helping the athlete to restore

\footnotetext{
a Corresponding author:

Mario Bizzini, PT, PhD

ORCID \# 0000-0002-4161-9163

Tel: +41 443857585

Email: mario.bizzini@sportfisio.ch
} 
Table 1. Graduated Return to Sport (RTS) Strategy of the Concussion in Sport Group (CISG)

\begin{tabular}{lll}
\hline Stage & Aim & Activity \\
\hline 1 & Symptom-limited activity & Daily activities that do not provoke symptoms \\
2 & Light aerobic exercise & Walking or stationary cycling at slow or medium pace. No resistance training \\
3 & Sport-specific exercise & Running or skating drills. No head impact activities \\
4 & Non-contact training drills & Harder training drills, e.g. passing drills. May start progressive resistance training \\
5 & Full contact practice & Following medical clearance, participate in normal training activities \\
6 & Return to sport & Normal game play \\
\hline
\end{tabular}

Consensus adapted from McCrory et al. ${ }^{2}$

their baseline function(s), but also requires working with them to improve, enhance and optimize performance.

\section{OPTIMIZING RETURN TO PERFORMANCE}

From a sports physical therapy and athletic trainer perspective, there is a need to be more actively involved in the RTS process rather than passively guiding the athlete.

While it is paramount to closely monitor SRC symptoms (i.e. before/during/after exercising), the inclusion of additional individual-adapted training activities can help the athlete in their "training to perform" at the different stages of the RTS program (Table 2). Table 2 outlines an example of a suitable RTS program for elite ice hockey players with SRC: the athlete should not only progress through the RTS stages, but also work on any deficits linked directly (i.e. balance abilities) and/or indirectly (i.e. reactive capacities) with the SRC.

In Stages 2 and 3 of the recommended program, the player is often supervised by the sports physical therapist or athletic trainer: while the focus is set on aerobic exercise (initially using a stationary bike or elliptical trainer to avoid neck/head impact) and initiating light resistance training, further elements can be added to facilitate player recovery. ${ }^{8-10}$ In the gym, the player may use skates and a stick for balance and coordination drills, and the slide board for (controlled) agility drills (Figures $\underline{1}, \underline{2}, \underline{3}$ ). While the player is still far away from the ice, the additional neuromuscular training-which should be role-specific depending on whether the affected athlete is a player or goalkeeper-enhances sport-specific reactive stabilization strategies and promotes a positive psychological attitude within the overall training regimen.

If symptoms persist for more than 10 to 14 days, the athlete should be referred to a healthcare professional with concussion management expertise. ${ }^{2,11}$ In particular, this is the case for players dealing with vestibular and/or oculomotor deficits (the topic of which is beyond the scope of this commentary), who often require comprehensive and targeted management. ${ }^{11-13}$

Deficient (or insufficient) activation of the deep cervical muscles is a common clinical observation in athletes after SRC, ${ }^{14}$ and it is therefore recommended to implement a neck stabilization/strengthening scheme in the RTS program usually during Stage 3 when SRC symptoms gradually resolve (Figure $\underline{4}, \underline{5}$ ). Despite the controversy surrounding

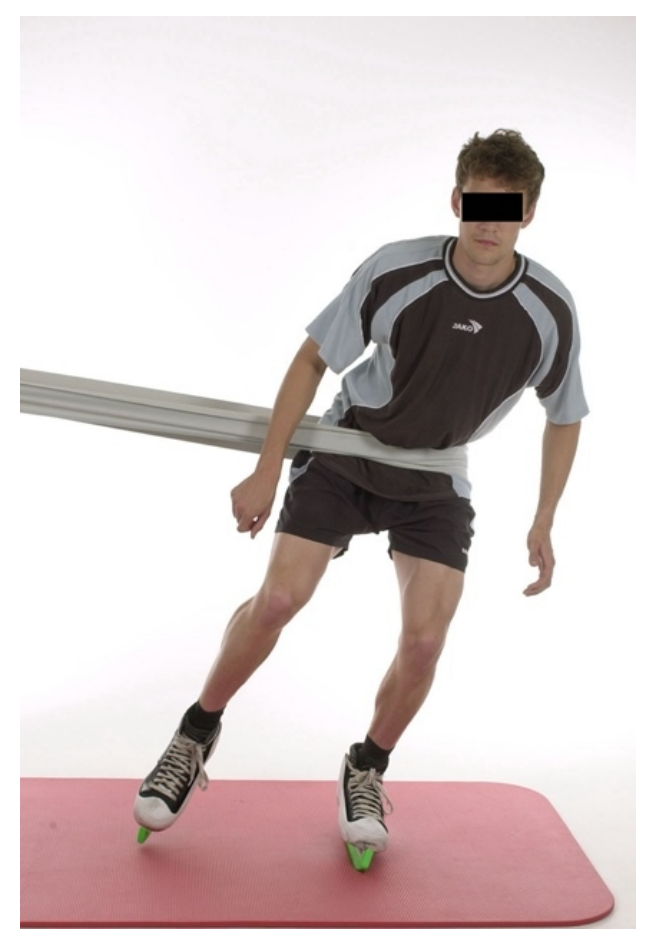

Figure 1. Reactive balance/stabilization on skates and against elastic resistance (duration/sets to be adapted)

this aspect of cervical involvement, a stable and stronger neck may contribute to a lower risk of further injury in athletes post-SRC. ${ }^{15-19}$

Within Stage 3, SRC players can begin with easy skating (while respecting the sub-symptom threshold) and before engaging in non-contact training (Stage 4.1), a basic set of on-ice drills is recommended (Stage 3.2). The assumption that a symptom-free player in the gym will remain symptom-free on the ice should be avoided; for example, if dizziness caused by head and/or body rotations has resolved with specific vestibular exercises, ${ }^{20}$ turning and pivoting on the ice may invoke renewed symptoms of head pressure or dizziness. Therefore, by allowing the player to proceed through a set of simple drills, where linear and circular/ rotational moves (forward and backward) with simple and dual tasks (i.e. adding puck control, focusing on a fixed/ moving target) are performed, the player is then able to feel 
Table 2. Graduated Return to Ice Hockey Program for Elite Players

\begin{tabular}{|c|c|c|}
\hline STAGE & $\operatorname{AIM}(S)$ & ACTIVITY \\
\hline 1 & $\begin{array}{l}\text { Symptom- } \\
\text { limited activity }\end{array}$ & Daily activities that do not provoke symptoms (e.g. 10 min of slow walking) \\
\hline 2 & $\begin{array}{l}\text { Light aerobic } \\
\text { exercises } \\
\text { (unspecific) }\end{array}$ & $\begin{array}{l}\text { a. Cardiovascular exercise on stationary bike; 25-40 min including warm up and cool down; controlled } \\
\text { activities, low to moderate intensity (ca. } 70 \% \mathrm{HR} \text { max); control for stable head/neck position while } \\
\text { exercising } \\
\text { b. Mobility/stretching, stabilization and controlled balance (double and single stance) exercises }\end{array}$ \\
\hline 3 & $\begin{array}{l}\text { 3.1 Aerobic } \\
\text { exercise } \\
\text { (progression) }\end{array}$ & $\begin{array}{l}\text { a. Introduce interval training on stationary bike (or elliptical; treadmill not first choice because of head/ } \\
\text { neck impacts); } 25-40 \text { min including warm up and cool down; controlled activities, moderate intensity } \\
\text { (ca. 70-80\% HR max); control for stable head/neck position while exercising } \\
\text { b. Body training (no resistance/light elastic resistance) } \\
\text { - Mobility and stretching exercises } \\
\text { - Neck stabilization exercises (no resistance) } \\
\text { - Trunk strength/stabilization exercises (no resistance; no explosive movements) } \\
\text { - Basic lower/upper extremities strength exercises (light elastic resistance) in the } 3 \text { planes } \\
\text { of movement } \\
\text { - Balance exercises (double and single stance) on unstable surfaces }\end{array}$ \\
\hline
\end{tabular}

3.2 Ice hockeyspecific exercises

No heavy resistance training.

a. Warm up -free skating- for $10 \mathrm{~min}$ at low/moderate intensity

b. Basics skating with rotation/changing of direction

- Skating forwards, backwards, sideways, stop \& go (not explosive)

- Skating in the face-off circle, figure of 8 between the two face-off circles

c. Technical training with the stick/puck (1:1)

- Basics: stickhandling, short/long passing; easy shooting on targets

No contact activities on ice.

For goalkeepers: controlled and non-explosive movements (T-glide, butterfly, half-butterfly, other); no shooting on goal

\begin{tabular}{|c|c|c|}
\hline 4.1 & $\begin{array}{l}\text { Non-contact ice } \\
\text { hockey training } \\
\text { drills }\end{array}$ & 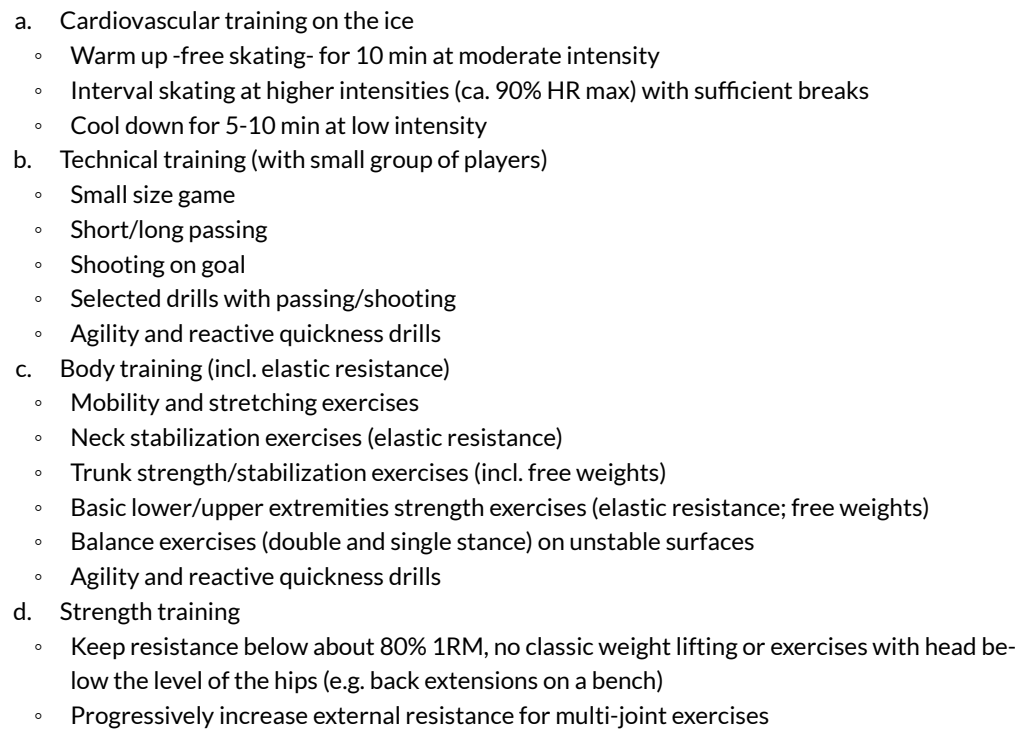 \\
\hline
\end{tabular}

No contact activities on ice.

For goalkeepers: controlled and explosive movements; shooting on goal 1:1 with a coach from different ranges (short to long) and angles (no slapshots)

\begin{tabular}{lll}
\hline 4.2 & $\begin{array}{l}\text { Ice hockey } \\
\text { training drills } \\
\text { with controlled } \\
\text { contact }\end{array}$ & $\begin{array}{l}\text { Participation in team training with controlled contact situations (checks on players, checks on } \\
\text { boards) } \\
\text { Progressively increase intensity in all training drills } \\
\text { For goalkeepers: all movements; drills with players shooting (incl. slapshots) on goal from } \\
\text { different angles }\end{array}$ \\
\hline 5 & $\begin{array}{l}\text { Full contact } \\
\text { practice (team }\end{array}$ & $\begin{array}{l}\text { Following medical clearance, participate in normal team training } \\
\text { a. Cardiovascular training: to be continued }\end{array}$ \\
\hline & \\
\hline
\end{tabular}


training) b. Body and strength training: regain usual routine training (unrestricted)

c. Assess and assure psychological readiness

$6 \quad$ Return to play Normal match play

$\mathrm{HR} \max =$ maximum heart rate, $\min =$ minutes, $\mathrm{RM}=$ repetition maximum

(and the coaches can observe) whether they are experiencing symptoms during these movements on the ice or not (Figure 6). The ability to master these drills on the ice without symptoms improves the player's confidence and raises the chances that they will be able to resume non-contact team practice efficiently.

Ice hockey is a fast-paced, contact team sport that requires high levels of reaction time and decision making, and athletes after SRC need to master motor and cognitive skills concurrently. Affected players usually experience difficulties with quickness and reactivity drills, which confirms recent findings on impaired reaction times during sportsrelated movement tasks. ${ }^{21,22}$ The inclusion of cognitive reactive agility drills with dual or multitasks (e.g. visuomotor reaction time training with the FITLIGHT ${ }^{\circledR}$ system) is therefore an important element in the "training to perform" part of Stage 4 and beyond (both in the gym and on ice). ${ }^{23,24}$ (Figure $\mathbf{7}, \underline{8}$ ) In addition to strength and conditioning training, these cognitive reactive agility drills are also necessary to minimize subsequent injury risk. ${ }^{25,26}$

At Stage 5 of our proposed RTS strategy, the player needs, on average, 4 to 5 full team training practice sessions (including unrestricted contact) before they can be considered completely mentally and physically ready for participation in competition games. As previously mentioned, this process is highly individual and should be based on both the athlete's response and staff judgement of the athlete's performance during training. The psychological readiness for competition is crucial, and the use of the Injury-Psychological Readiness to Return to Sport (I-PRSS) tool is helpful to assess the player's confidence in performing at the last stages of the RTS scheme. ${ }^{27,28}$ Neurocognitive testing (not discussed here) should also be used as part of the RTS decision-making process. ${ }^{11}$ Depending on the position, role and importance of the player within the team, the coaching staff often plan a number of games with increasing ice time (usually with the farm team) to allow the progressive return of the player to competition. With appropriate monitoring of the player's training progression and close interaction with the player, the medical and technical staff can best support the athlete in the "training to perform" stage of the RTS program, which should ideally be a shared decision-making process among these stakeholders. ${ }^{6}$ Ultimately, RTS after SRC should only occur with medical clearance from a licensed healthcare provider who is trained in the evaluation and management of concussion. ${ }^{13}$

\section{CONCLUSION}

Sport-related concussion is a severe and complex type of injury requiring specific rehabilitation management and a

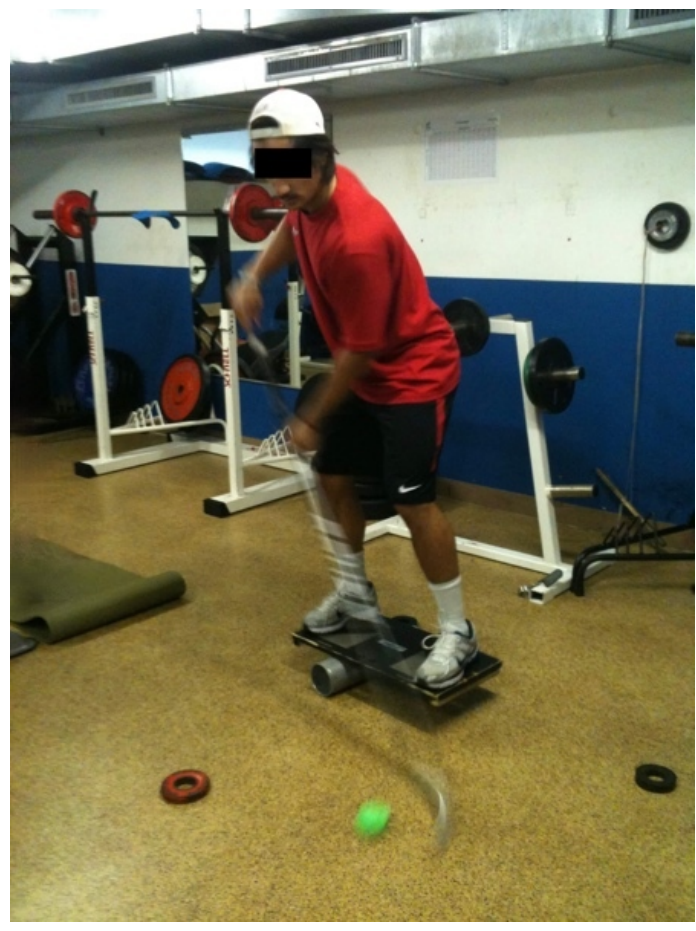

Figure 2. Balance/stabilization on board with stickhandling and moving the puck between two plates (duration/sets to be adapted)

RTS protocol following the CISG guidelines. While respecting these principles, the supporting team comprising a sports physical therapist and athletic trainer should work with the athlete to restore function as well as optimize and enhance their performance. The athlete is constantly supervised by the medical team during this process. Elite ice hockey players post-SRC should not only be symptom-free and able to participate in unrestricted practice and games, they should also be optimally ready at both physical and mental levels to perform with minimal re-injury risk. This clinical commentary has described a comprehensive RTS ice hockey program, which can help injured elite players in reaching this goal.

\section{ACKNOWLEDGEMENTS}

The author would like to acknowledge Nik Hess (athletic trainer at SCL Tigers, Langnau, Switzerland), Steven Lingenhag (athletic trainer at Hockey Club Davos, Davos, Switzerland) and Gilles Neuenschwander (physical therapist and athletic trainer at Hockey Club Ambrì Piotta, 
Quinto, Switzerland) for their invaluable cooperation over the years and assistance in the development of this proposed RTS protocol. Melissa Wilhelmi, $\mathrm{PhD}$ (medical writer at Schulthess Clinic, Zurich, Switzerland) participated in the copyediting and final proofreading of this manuscript.

Submitted: August 27, 2021 CST, Accepted: November 12, 2022 CST

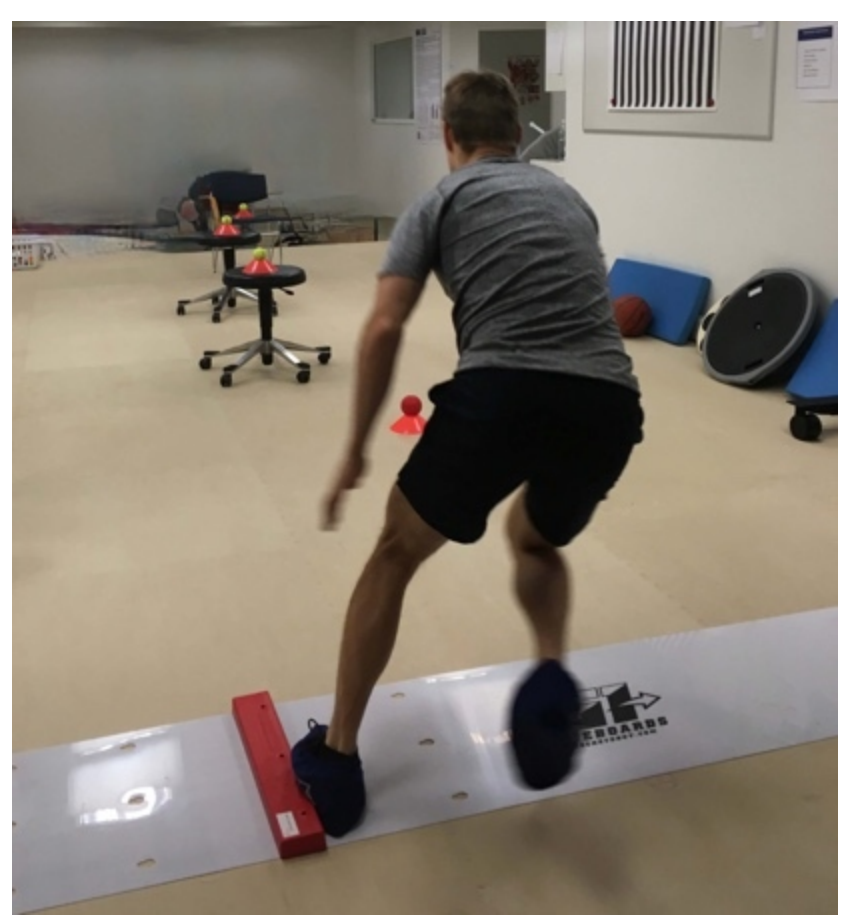

Figure 3. Moving on the slide board with an oculomotor task (fixing objects at various distances) (duration/sets to be adapted)

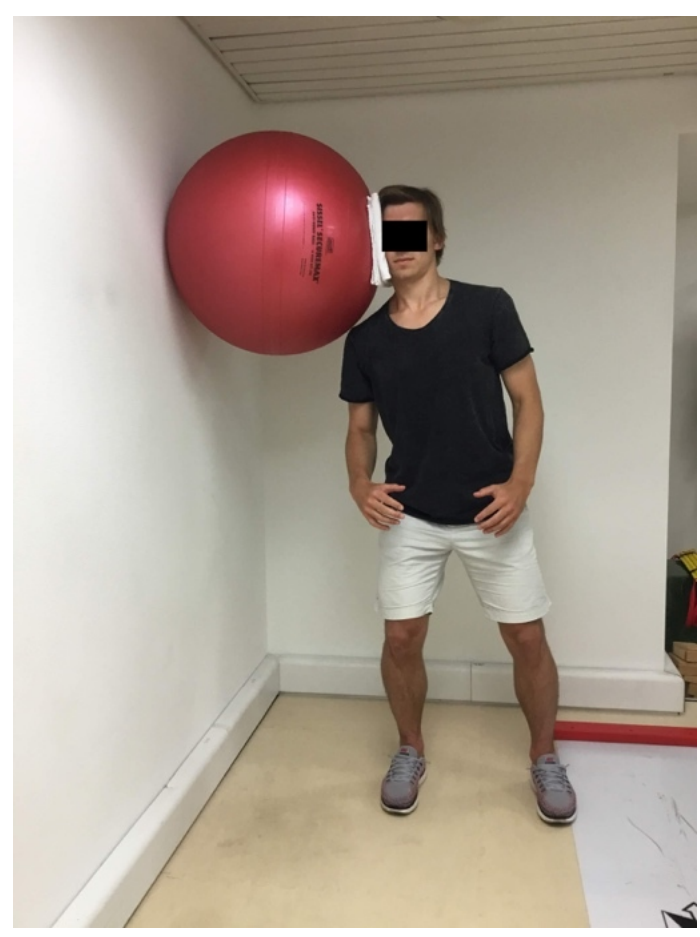

Figure 4. Isometric neck stabilization (lateral muscles) against a Swiss ball (duration/sets to be adapted) - Stage 3 (Table 2) 


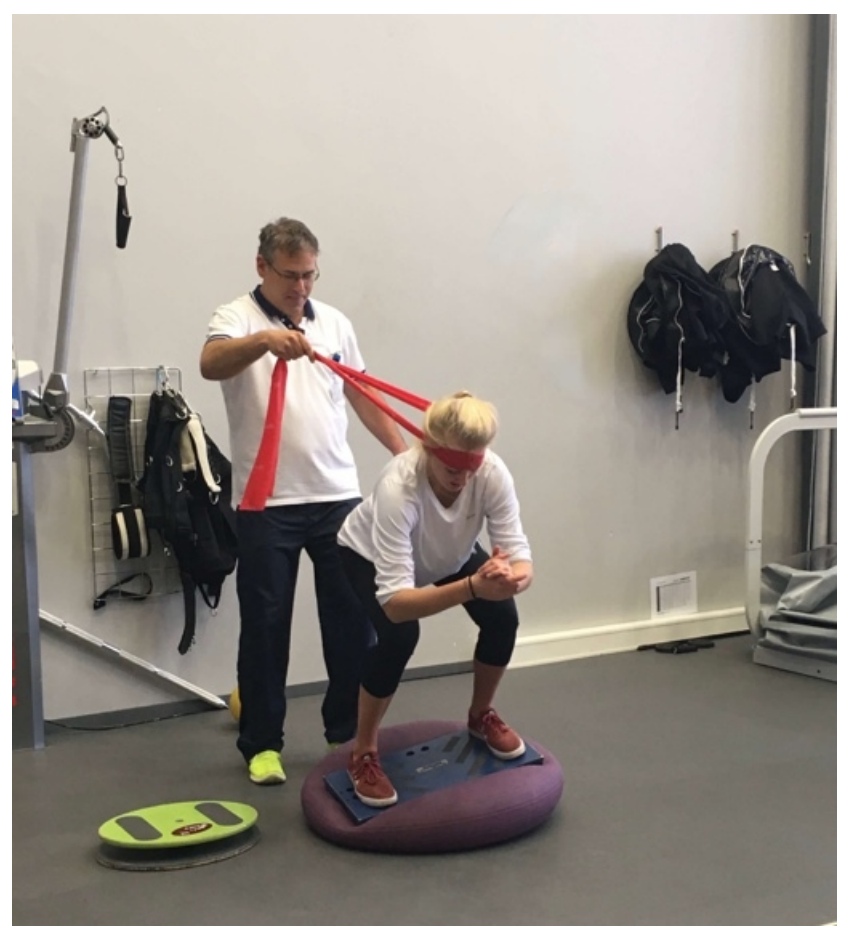

Figure 5. Reactive neck stabilization against elastic resistance, on an instable surface (duration/sets to be adapted) - Stage 4 (Table 2)

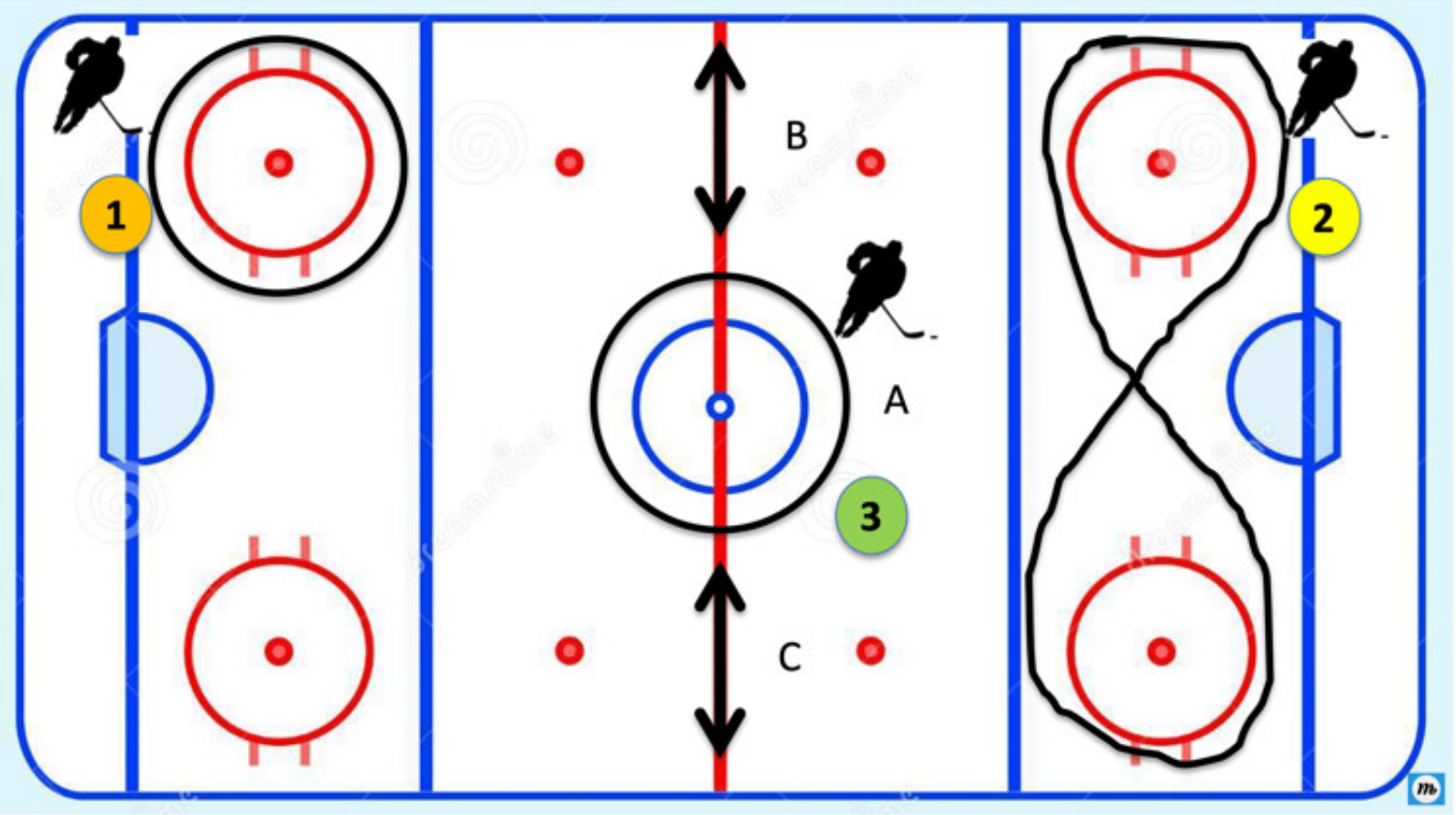

Figure 6. Scheme of basic moves on ice: 1) Bully circle, skating one/other directions; 20-30 sec per each direction; 2-3 sets with increased speed (stick only/ stick \& puck) 2) Figure 8 skating, $1 / 2$ forwards \& 1/2 backwards; 20-30 sec per each direction; 2-3 sets with increased speed (stick only/ stick and puck) 3) A: circling $2 \mathrm{x}=>$ B) accelerate to the boards, stop and back => A) + C) accelerate to boards, stop and back => continue (stick only) (duration/sets to be adapted) 


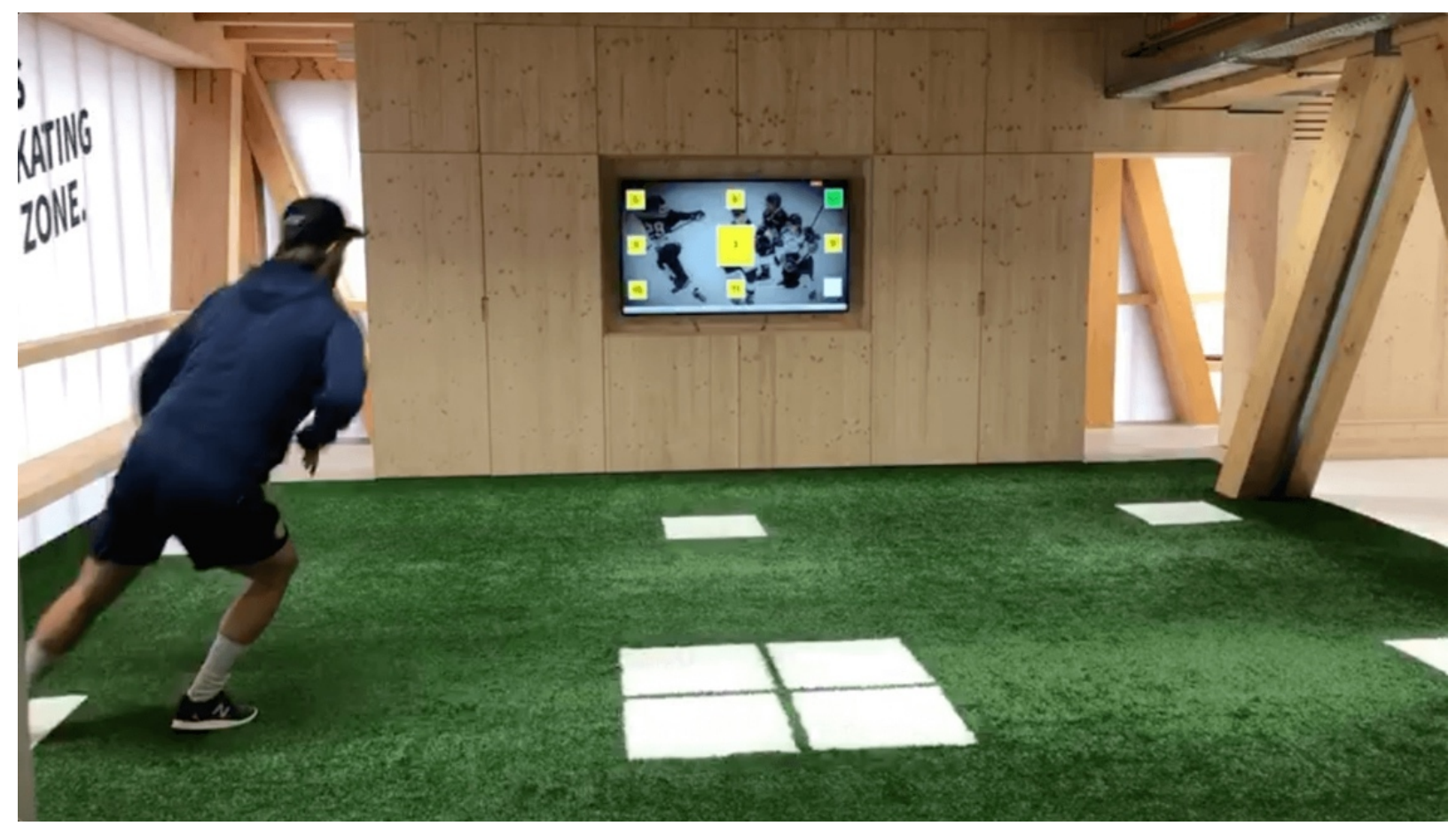

Figure 7. Visuomotor reactive training on the "Speed Court” (GlobalSpeed GmbH, Hemsbach, Germany). The player moves quickly form plate to plate reacting to the given signal on the screen placed on the wall in front of him. Drills of about 20-30-40 sec duration (sets to be adapted). Photo courtesy of Steven Lingenhag (Hockey Club Davos).

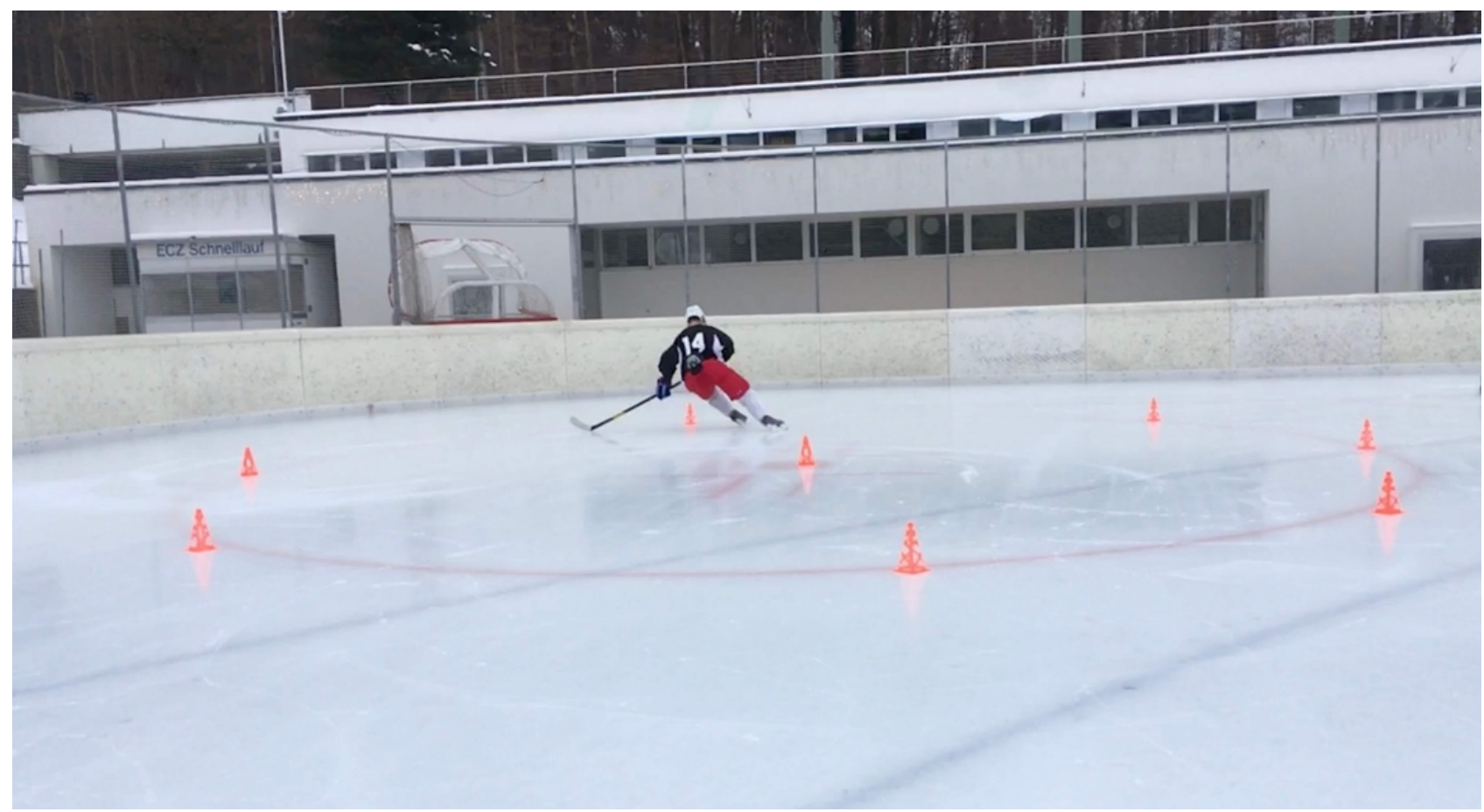

Figure 8. Several cones on the bully circle line and one in the middle. Player skates quickly and randomly between cones (stick/stick and puck). Progression: Fitlights are placed on the cones, and the player skates quicky to the cones reacting to the given light signal (stick only). Drills of about 20-30 sec duration (sets to be adapted) 
This is an open-access article distributed under the terms of the Creative Commons Attribution 4.0 International License (CCBY-NC-4.0). View this license's legal deed at https://creativecommons.org/licenses/by-nc/4.0 and legal code at https://creativecommons.org/licenses/by-nc/4.0/legalcode for more information. 


\section{REFERENCES}

1. Prien A, Grafe A, Rössler R, Junge A, Verhagen E. Epidemiology of head injuries focusing on concussions in team contact sports: a systematic review. Sports Med. 2018;48(4):953-969. doi:10.1007/s 40279-017-0854-4

2. McCrory P, Meeuwisse W, Dvořák J, et al. Consensus statement on concussion in sport-the 5(th) international conference on concussion in sport held in Berlin, October 2016. Br J Sports Med. 2017;51(11):838-847.

3. Feddermann - Demont N, Chiampas G, Cowie CM, et al. Recommendations for initial examination, differential diagnosis, and management of concussion and other head injuries in high - level football. Scand J Med Sci Sports.

2020;30(10):1846-1858. doi:10.1111/sms.13750

4. The NHL/NHLPA Concussion Evaluation and Management Protocol 2019-2020 season. Published online June 1, 2019. https://nhlpa.com/the-pa/pa-pro grams-and-partnerships/concussion-protocol-and-ed ucation

5. International Ice Hockey Federation concussion protocol. Published online September 5, 2019. http s://www.iihf.com/en/static/5070/medical

6. Ardern CL, Glasgow P, Schneiders A, et al. 2016 Consensus statement on return to sport from the First World Congress in Sports Physical Therapy, Bern. Br J Sports Med. 2016;50(14):853-864. doi:10.11 36/bjsports-2016-096278

7. Reinold MM. Performance physical therapy is sports physical therapy: why our profession needs to progress. Int J Sports Phys Ther. 2021;16(2). doi:10.26 603/001c.21556

8. Bizzini M, Gorelick M, Drobny T. Lateral meniscus repair in a professional ice hockey goaltender: a case report with a 5-year follow-up. J Orthop Sports Phys Ther. 2006;36(2):89-100. doi:10.2519/jospt.2006.3 $\underline{6.2 .89}$

9. Casartelli NC, Bizzini M, Maffiuletti NA, Lepers R, Leunig M. Rehabilitation and return to sport after bilateral open surgery for femoroacetabular impingement in a professional ice hockey player: A case report. Phys Ther Sport. 2015;16(2):193-201. do i:10.1016/i.ptsp.2014.08.002

10. Wolfinger CR, Davenport TE. Physical therapy management of ice hockey athletes: from the rink to the clinic and back. Int J Sports Phys Ther.

2016;11(3):482-495.
11. Collins MW, Kontos AP, Reynolds E, Murawski CD, $\mathrm{Fu}$ FH. A comprehensive, targeted approach to the clinical care of athletes following sport-related concussion. Knee Surg Sports Traumatol Arthrosc. 2014;22(2):235-246. doi:10.1007/s00167-013-2791-6

12. Schneider KJ, Leddy JJ, Guskiewicz KM, et al. Rest and treatment/rehabilitation following sport-related concussion: a systematic review. Br J Sports Med. 2017;51(12):930-934. doi:10.1136/bjsports-2016-0974 $\underline{75}$

13. Harmon KG, Clugston JR, Dec K, et al. American Medical Society for sports medicine position statement on concussion in sport. Clin J Sport Med. 2019;29(2):87-100. doi:10.1097/jsm.00000000000007 $\underline{20}$

14. Jull GA, O’Leary SP, Falla DL. Clinical assessment of the deep cervical flexor muscles: the craniocervical flexion test. J Manipulative Physiol Ther. 2008;31(7):525-533. doi:10.1016/i.jmpt.2008.08.003

15. Hrysomallis C. Neck muscular strength, training, performance and sport injury risk: a review. Sports Med. 2016;46(8):1111-1124. doi:10.1007/s40279-01 6-0490-4

16. Streifer M, Brown AM, Porfido T, Anderson EZ, Buckman JF, Esopenko C. The potential role of the cervical spine in sports-related concussion: clinical perspectives and considerations for risk reduction. J Orthop Sports Phys Ther. 2019;49(3):202-208. doi:10.2 519/jospt.2019.8582

17. Smith AM, Alford PA, Aubry M, et al. Proceedings from the Ice Hockey Summit III: Action on Concussion. Curr Sports Med Rep. 2019;18(1):23-34. ㅁ oi:10.1249/jsr.0000000000000557

18. Daly E, Pearce AJ, Ryan L. A systematic review of strength and conditioning protocols for improving neck strength and reducing concussion incidence and impact injury risk in collision sports; is there evidence? J Funct Morphol Kinesio. 2021;6(1):8. doi:1 $\underline{0.3390 / j \mathrm{fmk} 6010008}$

19. Elliott J, Heron N, Versteegh T, et al. Injury reduction programs for reducing the incidence of sport-related head and neck injuries including concussion: A systematic review. Sports Med. Published online June 18, 2021:40279-40021. doi:10.1 007/s40279-021-01501-1 
20. Schneider KJ, Meeuwisse WH, Nettel-Aguirre A, et al. Cervicovestibular rehabilitation in sport-related concussion: a randomised controlled trial. Br J Sports Med. 2014;48(17):1294-1298. doi:10.1136/bjsports-20 13-093267

21. Lynall RC, Blackburn JT, Guskiewicz KM, Marshall SW, Plummer P, Mihalik JP. Reaction time and joint kinematics during functional movement in recently concussed individuals. Arch Phys Med Rehabil. 2018;99(5):880-886. doi:10.1016/j.apmr.2017.12.011

22. Howell DR, Lynall RC, Buckley TA, Herman DC. Neuromuscular control deficits and the risk of subsequent injury after a concussion: a scoping review. Sports Med. 2018;48(5):1097-1115. doi:10.100 7/s40279-018-0871-y

23. Avedesian JM, Forbes W, Covassin T, Dufek JS. Influence of cognitive performance on musculoskeletal injury risk: A systematic review. Am J Sports Med. Published online March 19, 2021:036354652199808. doi:10.1177/03635465219980 81
24. Wilkerson GB, Grooms DR, Acocello SN. Neuromechanical considerations for postconcussion musculoskeletal injury risk management. Curr Sports Med Rep. 2017;16(6):419-427. doi:10.1249/jsr.0000000 $\underline{000000430}$

25. McPherson AL, Nagai T, Webster KE, Hewett TE. Musculoskeletal injury risk after sport-related concussion: a systematic review and meta-analysis. Am J Sports Med. 2019;47(7):1754-1762. doi:10.1177/0 $\underline{363546518785901}$

26. Chmielewski TL, Tatman J, Suzuki S, et al. Impaired motor control after sport-related concussion could increase risk for musculoskeletal injury: Implications for clinical management and rehabilitation. J Sport Health Sci. 2021;10(2):154-161. doi:10.1016/j.jshs.2020.11.005

27. Glazer DD. Development and preliminary validation of the Injury-Psychological Readiness to Return to Sport (I-PRRS) scale. J Athl Train. 2009;44(2):185-189. doi:10.4085/1062-6050-44.2.185

28. Caron JG, Bloom GA, Podlog LW. Are athletes psychologically ready for sport following a concussion? Br J Sports Med. 2018;52(1):1-2. doi:10.1 136/bjsports-2017-098319 